\title{
C-07
}

\section{OPTIMIZACIÓN DE LA PRODUCTIVIDAD DEL AGUA EN ZONAS REGABLES MEDIANTE UNA HERRAMIENTA DE SIMULACIÓN DEL SISTEMA HIDRÁULICO, AGRARIO Y ECONÓMICO}

\author{
Elmalki, E. ${ }^{1}$, Playán, E. ${ }^{2}$, Gallinat, J. $^{3}$ y Zapata, N. $^{4}$
}

\begin{abstract}
${ }^{1}$ Personal Investigador en Formación. Depto. Suelo y Agua, Estación Experimental Aula Dei (EEADCSIC). Avda. Montañana 1005, 50059, Zaragoza. E-mail: hmalki@eead.csic.es

${ }^{2}$ Profesor de Investigación Depto. Suelo y Agua, Estación Experimental Aula Dei (EEAD-CSIC). Avda. Montañana 1005, 50059, Zaragoza. E-mail: enrique.playan@csic.es

${ }^{3}$ Ingeniero en la empresa CINGRAL S.L., C/Santa Cruz, 8, bajo. 50003, Zaragoza. Email: igallinat@cingral.es.

${ }^{4}$ Científico Titular, Depto. Suelo y Agua, Estación Experimental Aula Dei (EEAD-CSIC). Avda. Montañana 1005, 50059, Zaragoza. E-mail: v.zapata@csic.es
\end{abstract}

\section{Resumen}

Este trabajo presenta una aplicación de la herramienta de simulación CINTEGRAL que permite adaptar el diseño de la red de riego a la realidad agraria local de las zonas regables (meteorología, suelos, cultivos y productividad global). Se estudian las condiciones locales (meteorología, suelos y cultivos) de una zona regable del Valle Medio del Ebro cuya red se ha diseñado de forma estándar. Se analiza el efecto de la variabilidad meteorológica y de diferentes alternativas de gestión del riego sobre la productividad global de la zona. Se discuten las diferencias en producción y productividad global de la zona bajo tres alternativas de gestión del riego: 1) gestión a la demanda, 2) gestión en turnos de riego coincidentes con pisos de presión, y 3) gestión en dos redes independientes. Los resultados obtenidos muestran un efecto claro de la variabilidad meteorológica sobre los ingresos brutos, ingresos netos y la factura eléctrica. La gestión del riego en turnos ajustados en función de la presión requerida permite ahorrar el $22 \%$ en la factura eléctrica respecto de la gestión del riego a la demanda. Sin embargo, las producciones se ven afectadas de forma muy notable, reduciendo notablemente la productividad de la zona. Para mantener la productividad con un riego a turnos es necesario realizar modificaciones en el diseño original de la red. Por lo tanto, en esta zona la gestión en turnos coincidentes con pisos de presión no mejora los ingresos netos. La división de la red global en dos sub redes independientes de riego reduce la factura eléctrica en un $20 \%$ y además mantiene los niveles de producción, por lo que los ingresos netos se incrementan en un $9 \%$.

\section{Introducción}

El diseño de los sistemas colectivos de riego por aspersión en cobertura total está basado en varios supuestos: 1) Una hipótesis de necesidades hídricas de la alternativa de cultivos prevista; 2) Una hipótesis sobre el tiempo disponible para el riego, expresado en porcentaje; 3) Una hipótesis de presión mínima necesaria a la salida de hidrante; 4) Una hipótesis sobre el modelo de reparto de agua; y 5) Una hipótesis de simultaneidad de la operación de la red.

Esta metodología ha llevado al diseño de las redes de riego que permitieran un funcionamiento compatible con las condiciones supuestas aguas abajo. Este funcionamiento resulta en la práctica en un nivel de garantía muy alto, que optimiza la red de forma hidráulica y que permite minimizar el coste de inversión de la red o el coste energético. Sin embargo, esta metodología arrastra carencias importantes derivadas de un proceso de diseño de arriba (el punto de captación) a abajo (el sistema de riego en parcela). Ignorar en el diseño las condiciones aguas abajo del hidrante ha llevado a que las redes las diseñen los 
estudios de ingeniería, mientras que en los sistemas de riego en parcela los instaladores instalen soluciones estándar, no adaptadas a las situaciones particulares de los cultivos, del medio o del suministro de agua. El desacople entre la red y el sistema de riego en parcela con frecuencia ha llevado a sistemas sobredimensionados en capacidad de conducción y en necesidades de presión.

En los últimos años se ha producido un cambio sustancial en las condiciones de los proyectos de modernización de regadíos y de nuevos regadíos. Así pues, en la actualidad: 1) Es preciso minimizar el coste de las nuevas instalaciones, tanto en inversión como en explotación (coste de energía); y 2) En redes con bombeos es preciso contar con sistemas de demanda negociada que permitan optimizar no sólo el consumo de energía sino su coste.

Estas circunstancias se combinan con progresos relevantes en investigación sobre sistemas de riego por aspersión. Uno de estos desarrollos en investigación es CINTEGRAL (Zapata et al. 2015) una herramienta de diseño de zonas regables que acopla desarrollos ya publicados en todos los procesos que participan en el riego por aspersión de una zona colectiva: hidráulica de redes presurizadas (basadas en EPANET, Rossman et al. 1994), distribución de agua desde el emisor hasta el cultivo basada en la teoría balística (Fukui et al. 1980) y aprovechamiento del agua por el cultivo, basado en modelos de cultivos como CropWat (Smith 1992).

El objetivo de este trabajo es evaluar sobre una zona regable del Valle Medio del Ebro cuya red se ha diseñado de forma estándar, el efecto que tendrá sobre la productividad de la zona la variabilidad meteorológica local así como diferentes alternativas de gestión del riego. Así se estudiarán tres formas alternativas de gestión del riego: 1) Gestión a la demanda (GRD); 2) Gestión del riego a turnos coincidentes con pisos de presión manteniendo el diseño original (GRT $T_{D O}$ ) o con pequeñas modificaciones (GRT $\left.T_{D M}\right)$; y 3) Gestión del riego en dos redes de riego independientes gestionadas a la demanda y coincidentes con las dos zonas de diferentes necesidades de presión (GR2R).

\section{Material y métodos}

\subsection{Herramienta CINTEGRAL}

La herramienta CINTEGRAL ha sido creada de forma cooperativa por investigadores de la EEAD-CSIC y por la empresa CINGRAL S.L. La herramienta permite realizar el diseño de una zona regable adaptándose a los suelos, la meteorología y la agronomía, así como a los precios de las cosechas y de los insumos de producción (entre ellos el agua y la energía), es decir, considerando la realidad agraria local de forma global.

CINTEGRAL engloba un conjunto de modelos y programas ya desarrollados en trabajos anteriores, los más importantes son los siguientes:

1. El modelo de red parcelaria y de la red general colectiva, para ello se utilizó el programa ADOR-Red desarrollado y presentado en Zapata et al. (2009), al que se le incorporó el módulo de simulación hidráulica desarrollado a partir de EPANET (Rossman et al. 1994).

2. El modelo de reparto de agua desde el emisor hasta la planta en riego por aspersión ADOR-Aspersión desarrollado y presentado en Playán et al. (2006) para cobertura total de aspersión.

3. El modelo ADOR-Cultivo desarrollado y presentado en Dechmi et al. (2004a). Se adaptó a los cultivos más importantes de la zona. Se revisaron y ajustaron las ecuaciones de cálculo de las necesidades hídricas de los cultivos y de su respuesta al estrés hídrico.

4. Un módulo de optimización que analiza el funcionamiento de la zona en términos de productividad global y que permite optimizar la contratación de potencia.

\subsubsection{Input del programa}

Para su funcionamiento el programa requiere numerosos datos de entrada que sintetizan las condiciones locales de la zona de estudio. A continuación se resumen los datos de entrada necesarios:

1. El diseño hidráulico de la red general y algunos datos sobre la red parcelaria. Las redes parcelarias no son necesarias ya que no se realiza una simulación hidráulica de cada una de 
estas redes, sino que se simplifican. Para ello se requieren datos básicos sobre la parcela, tales como la disposición y distanciamiento entre aspersores, o el número y tamaño de las boquillas de los aspersores. Con el caudal del hidrante disponible en la red general, la superficie de la parcela y el caudal del aspersor se calcula el número probable de sectores o posturas de riego de la parcela.

2. El cultivo y el/los suelos de las parcelas. Esta asignación puede ser manual si se dispone de un mapa de suelos y cultivos de la zona, o se puede hacer una asignación automática proporcionándole a la herramienta el porcentaje en superficie de cada cultivo o tipo de suelo. La asignación automática de cultivo y suelo a cada parcela se realiza así de forma aleatoria.

3. Los datos eléctricos: Potencia contratada en cada periodo tarifario, así como el precio de la potencia y el de la energía de cada periodo. La herramienta permite realizar una optimización de la potencia a contratar en cada periodo tarifario a partir de la alternativa de cultivos de la zona y de una serie meteorológica local.

4. Los datos de gestión del riego: si queremos limitar los riegos por motivos de calidad mínima (uniformidad de riego mínima, eficiencia mínima) o de duración.

5. Los datos meteorológicos locales. Para ello la herramienta proporciona una base de datos de las estaciones agrometeorológicas de la red SIAR de Aragón que disponen de todos los datos necesarios para realizar la simulación. De esta forma el usuario sólo tiene que seleccionar la estación más cercana de la zona de estudio, así como el año de datos en el que quiere realizar el análisis. La base de datos de estaciones meteorológicas puede ampliarse con estaciones de otras zonas de España con disponibilidad de datos diarios y semihorarios.

6. Módulo económico. Por un lado se incorporan los datos globales de inversión, el de la red colectiva y el de las parcelarias (se totaliza el coste de las redes parcelarias), así como las condiciones de financiación para poder obtener la amortización anual. Además, para calcular los ingresos netos por cosecha necesitamos incorporar para cada cultivo: los costes de producción (sin incluir el coste del riego que se calculará en el proceso), la producción máxima zonal (el programa calcula la producción como un porcentaje respecto al máximo) y los ingresos por venta de cosecha.

\subsubsection{Output del programa}

Los resultados que proporciona la herramienta responden a diferentes escalas espaciales, desde el sector o postura de riego de una parcela, pasando por la parcela, el hidrante hasta la zona regable. Para cada elemento de la escala espacial el programa proporciona resultados de la evapotranspiración potencial y la real, el volumen de riego bruto y el neto, la uniformidad y eficiencia de riego estacional, la producción, el coste energético, los ingresos brutos y los ingresos netos. Para visualizar estos resultados, CINTEGRAL proporciona dos opciones:

1. Visualización espacial. Para ello es necesario incorporar una cobertura GIS de los lotes regados por cada hidrante. En la tabla asociada a dicha cobertura GIS debe de aparecer una columna llamada "contenido" con los nombres de los hidrantes tal y como aparecen en el archivo de la red hidráulica. Con la cobertura GIS como fondo pueden graficarse los resultados obtenidos a escala de hidrante, como por ejemplo, el margen neto, la producción media, fallos de red (fallos por insuficiencia de presión), fallos debidos a la falta de energía, la dosis de riego, etc.

2. Visualización en forma de tablas y gráficos. Los resultados se presentan para diferentes escalas espaciales (toda la red, por hidrante, por parcela, por cultivo por sector). Se pueden comparar gráficamente diferentes simulaciones en términos de producción media, eficiencia y uniformidad de riego estacional, fallos de la red y de energía, volumen de riego en cada periodo, coste de agua, margen neto, etc.

\subsection{Zona de estudio}

Para el estudio se ha seleccionado una comunidad de regantes (CR) del Valle Medio del Ebro. Dicha comunidad tiene una superficie regable de 3644 ha, repartida en 273 hidrantes. 
Se utilizó la red SIAR (Sistema de Información Agroclimática para el Regadío) para la obtención de los datos. Dicha red proporciona datos semihorarios y diarios en todas sus estaciones. La estación más representativa de la zona de estudio es la de Grañén. En dicha estación se dispone de datos desde el año 2004 hasta 2014.

La red general de la CR se diseñó con un programa de diseño de redes presurizadas. Las redes parcelarias todavía no están diseñadas, por lo que se realizó un pre-diseño a partir del parcelario de la zona en formato GIS y de los datos de los hidrantes (caudal y presión) resultantes del diseño de la red general. Con el programa GVGIS, se delimitaron la superficie y el número de parcelas regadas por cada hidrante. Se supuso un diseño homogéneo de las parcelas con una disposición de los aspersores en tresbolillo a $18 \mathrm{~m}$ entre filas y $18 \mathrm{~m}$ entre aspersores (T18x18), muy común en zonas de riego de cultivos extensivos, y con aspersores de impacto de doble boquilla $(4,4 \mathrm{~mm}$ y $2,4 \mathrm{~mm})$. El diseño original de la red general se realizó para asegurar una presión en boquilla de $350 \mathrm{kPa}$. Sin embargo dicha presión resulta elevada para los costes energéticos actuales. Se realizó un segundo pre-diseño de las parcelas asegurando una presión en boquilla de $300 \mathrm{kPa}$.

A partir del mapa de suelos de los municipios Barbués y Torres de Barbués publicado por Nogués (2002) y utilizando la herramienta de análisis espacial GVSIG, se asignaron las características del suelo de las 3155 ha regadas. Solamente 1880 ha de la zona regable están incluidas en el mapa de suelos presentado por Nogués (2002). Para la superficie de la que no se disponía de mapa de suelos, se realizó una asignación aleatoria de los suelos, conservando los porcentajes del mapa original. Los parámetros del suelo que nos interesa establecer son aquellos más directamente relacionados con el riego: la profundidad efectiva y la capacidad de retención de agua (CRA).

En el mapa de Nogués (2002) la profundidad del suelo se clasifica en 4 clases, desde poco profundo $(30 \mathrm{~cm})$ a muy profundo $(140 \mathrm{~cm})$. En cuanto a la CRA se clasifica en cinco niveles, de muy baja (inferior a $64 \mathrm{~mm}$ en 1,5 m) a muy alta (superior a $250 \mathrm{~mm}$ en 1,5 m). Los datos de CRA se transformaron a $\mathrm{mm} / \mathrm{m}$ (unidades que utiliza la herramienta CINTEGRAL) y se atribuyó a cada parcela el tipo de suelo que le correspondía.

El diseño de la red general de riego se realizó para la alternativa de cultivos extensivos (\% de superficie cultivada de cada uno) que se presenta en la Tabla 1. En la misma también se presentan los datos económicos requeridos por el programa, como son: 1) los gastos de producción, incluyendo los costes de la semilla, la siembra, los fertilizantes, tratamientos fitosanitarios y sus aplicaciones, así como los de otras labores del cultivo incluida la cosecha (no se incluyen los costes del agua y la energía asociada, pues estos se calculan con la herramienta); 2) el rendimiento máximo de la zona, 3) el precio de venta de la cosecha.

Tabla 1. Alternativa de cultivos para la que se realizó el diseño de la zona regable. Se incluyen los gastos de producción, el rendimiento máximo esperable en la zona y el precio de venta.

\begin{tabular}{lcccc}
\hline Cultivo & $\begin{array}{c}\text { Porcentaje } \\
(\%)\end{array}$ & Gastos $€ /$ ha & $\begin{array}{c}\text { Rendimient } \\
\text { o Kg/ha }\end{array}$ & $\begin{array}{c}\text { Precios } \\
€ / \text { Tonelada }\end{array}$ \\
\hline Alfalfa & 40 & 1500 & 18000 & 130 \\
Maíz & 40 & 1500 & 14000 & 170 \\
Cebada & 10 & 450 & 4500 & 175 \\
Trigo & 10 & 500 & 4500 & 200 \\
\hline
\end{tabular}

\subsection{Análisis de alternativas de gestión y diseño de la zona regable}

\subsubsection{Gestión a la demanda}

Se plantea el análisis del funcionamiento de la red bajo las condiciones de diseño original. Dicho diseño se realizó para gestionar el riego a la demanda, GRD. La fiabilidad del diseño se analiza ante diferentes condiciones meteorológicas (serie meteorológica 2004-2014), en términos de productividad global de la zona. En principio se analizan los ingresos netos que resultan de descontar a los ingresos brutos los costes de producción y de aplicación del 
agua de riego (factura eléctrica). También se analiza la producción de los cultivos y los parámetros de la calidad de riego (uniformidad y eficiencia de riego), así como el consumo de agua.

\subsubsection{Gestión en turnos de riego}

Trabajos recientes indican que mejoras en la gestión de riego - como el riego por turnos en pisos de presión - pueden reducir los consumos energéticos hasta un 20\% (Carrillo-Cobo et al. 2011; Fernández-García et al. 2014a). En este trabajo se propone el análisis de la gestión del riego a turnos sobre el diseño original de la red (GRT $\mathrm{DO}_{\mathrm{DO}}$ ). El sistema a turnos se ha basado en asignar a cada turno parcelas con necesidades homogéneas de presión (pisos de presión). El análisis de las cotas y las necesidades de presión de cada hidrante permitió identificar dos zonas diferenciadas en cuanto a necesidades de presión. Así se identificaron: un turno o zona alta en el que se permitirá regar solo en las horas de energía barata (P6), y un turno o zona baja en el que se podrá regar en cualquier momento del día. Los dos turnos tienen aproximadamente la misma superficie.

La estación de bombeo se programa para que en los periodos de tarifa barata (P6) en los que solo riega el turno de necesidades altas de presión, la altura de bombeo sea igual a la de la red original, $63 \mathrm{~m}$, mientras que en el resto de periodos tarifarios, de P5 a P1, la altura de bombeo se regulará a $43 \mathrm{~m}$. Esta diferencia en la altura de bombeo afecta de igual forma a la potencia específica, pasando de $0,27 \mathrm{kWh} \cdot \mathrm{m}^{-3}$ cuando se bombea en P6 a 0,18 kWh.m $\mathrm{m}^{-3}$ cuando bombeamos en cualquier otro periodo tarifario. Se analizó el funcionamiento de la red para los dos turnos establecidos manteniendo el diseño original de la red y surgieron problemas. El diseño original se realizó para gestionar el riego a la demanda, es decir, de forma que existiera libre disponibilidad de apertura de los hidrantes en las condiciones de caudal y presión preestablecidas. Regar en turnos organizados en la forma establecida significa que una serie de hidrantes (los situados en la zona alta o turno alto) solo pueden regar en periodos de energía barata, lo que reduce su tiempo disponible para riego respecto para el que fueron diseñados. Este hecho puede acarrear una serie de carencias como que el hidrante no sea capaz de aplicar el riego necesario en el momento adecuado y la producción se resienta. Para evitar estas deficiencias se realizaron pequeños cambios en el diseño de la red de riego original, incrementando el caudal de algunos de estos hidrantes situados en la zona alta así como los diámetros de las tuberías que los alimentan. Esta nueva alternativa de gestión de riego a turnos con diseño modificado (GRT $T_{\mathrm{DM}}$ ) se comparó con la GRT $T_{\mathrm{DO}}$ y con la GRD. Las comparaciones se realizaron en términos de producción de los cultivos, de costes eléctricos y de ingresos netos.

\subsubsection{Gestión en dos redes independientes}

La CR es muy grande y heterogénea, con un desnivel máximo muy importante (52 m entre la parcela de mayor elevación respecto a la de menor). Estas diferencias tan notables parecen indicar la conveniencia de analizar el funcionamiento de la zona regable dividiendo la zona en dos subzonas con dos redes independientes (GR2R: Gestión de Riego en dos Redes). Los nuevos diseños se realizaron mediante las herramientas GVSIG y GESTAR. La distribución de los porcentajes de superficie de cultivos en ambas redes fue la misma que la alternativa original (Tabla 1). La distribución en dos subzonas fue la misma que la realizada para dividir la zona en dos turnos de riego: zona alta (mayores requerimientos de presión) y zona baja (menores requerimientos de presión). Las comparaciones GRD, GRT $T_{\mathrm{DO}}$, $\mathrm{GRT}_{\mathrm{DM}}$ y GR2R se realizaron en términos de coste eléctrico, ingresos brutos (producciones), ingresos netos y productividad global de la zona.

\section{Resultados y discusión}

\subsection{Herramienta CINTEGRAL}

La Figura 1 muestra un ejemplo de los datos de entrada y de salida de la herramienta CINTEGRAL. En la Figura 1a se presentan como ejemplo de datos de entrada la pantalla de datos de hidrantes y de usos. De forma automática y a partir del diseño de la red general se 
completan una serie de datos generales (como superficie y caudal del hidrante) y se les asigna una parcela y un uso. Estos datos asignados de forma automática pueden editarse y modificarse manualmente. En la Figura 1b se muestra un ejemplo de la visualización espacial de los resultados obtenidos en cuanto a producción media por hidrante. Si se hace un clic sobre una parcela salen los datos y resultados numéricos de dicha parcela.

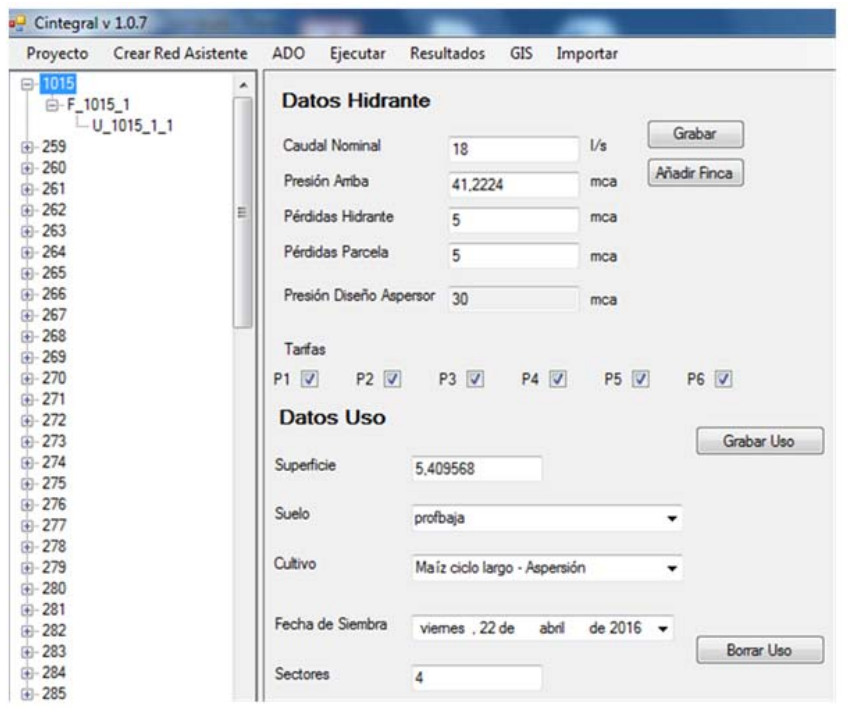

(a)

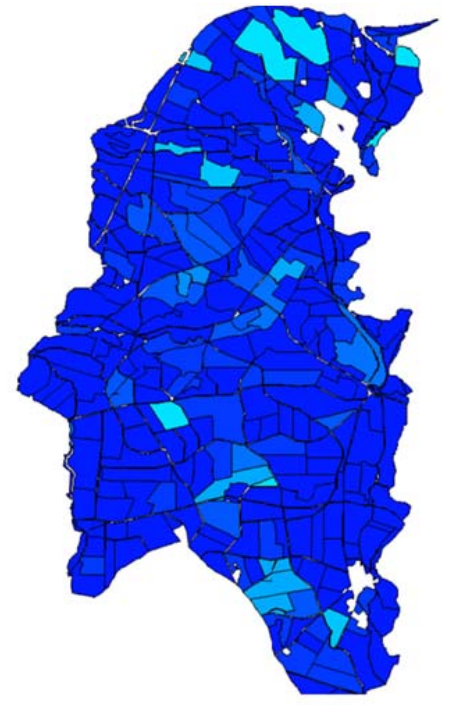

(b)

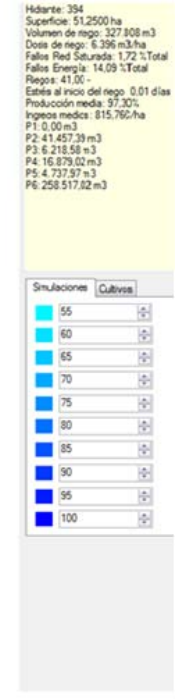

Figura 1. Ejemplo de datos de entrada (a) y de salida (b) de la herramienta CINTEGRAL.

\subsection{La zona de estudio}

La evapotranspiración de referencia $\left(\mathrm{ET}_{0}\right)$ presentó una media anual de $1205 \mathrm{~mm}$ año-1 con un CV de 3,8\%, un máximo de $1286 \mathrm{~mm}_{\text {año }}{ }^{-1}$, registrado en el año 2012, y un mínimo de $1119 \mathrm{~mm}$ año-1, en el año 2008. La ET 0 mensual máxima fue de $198 \mathrm{~mm}$, que se produce todos los años en el mes de julio. La ET $\mathrm{ET}_{0}$ más baja fue de $25 \mathrm{~mm}$, que se produce en todos los años en el mes de diciembre.

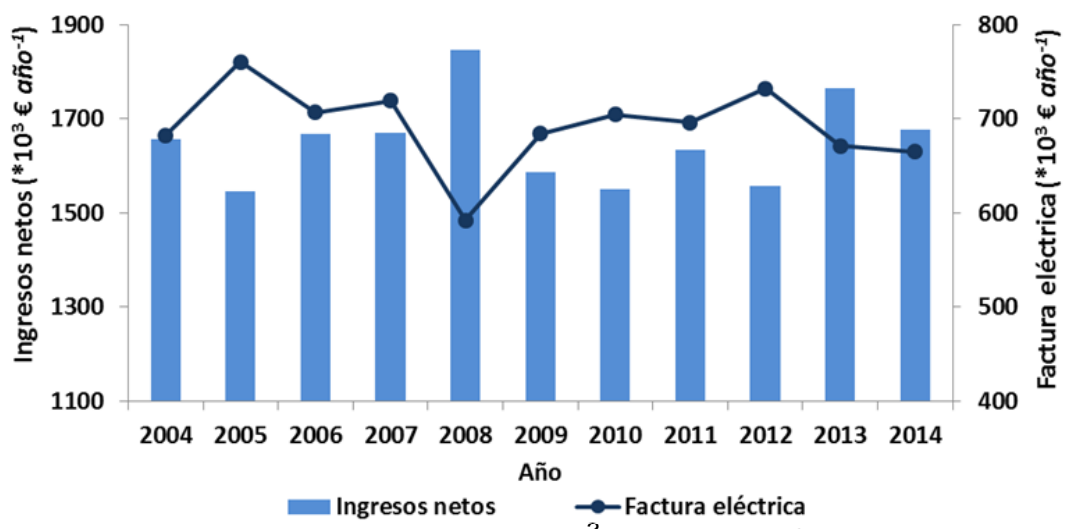

Figura 2. Ingresos netos y factura eléctrica en ${ }^{\star} 10^{3} €$ por año, GRD.

Se analizó el efecto de la variabilidad interanual de la meteorología sobre el funcionamiento de la red de riego a la demanda asegurando una presión de $300 \mathrm{kPa}$ en boquilla. La alternativa de cultivos presentada en la Tabla 1 y la serie meteorológica disponible se utilizaron para optimizar la potencia a contratar en cada uno de los tramos tarifarios y años meteorológicos. Para ello se utilizó el módulo de optimización descrito en la herramienta CINTEGRAL. Los resultados de ingresos netos y factura eléctrica para la serie meteorológica analizada se presentan en la Figura 2. La media interanual de los ingresos netos fue de 2,342 M€, con un CV de 2,6\%. El coste de la factura eléctrica medio fue de 
0,692 M€, con un CV de 6,2\%. El año de menor coste fue $2008(0,592 \mathrm{M})$ ) y el de mayor coste $(0,760 \mathrm{M} €)$ fue 2005 . En el año 2008 se obtuvieron los mayores ingresos netos de la serie analizada $(1,850 \mathrm{M} €)$, mientras que en el 2005 se obtuvieron los menores ingresos netos $(1,545 \mathrm{M})$. Parte de la diferencia se debe a los costes de la factura eléctrica, que en 2008 fueron los más bajos y en 2005 los más elevados de la serie estudiada. La meteorología tiene mucho que decir sobre estos resultados, ya que 2008 fue un año de precipitaciones por encima de la media (especialmente en los meses de verano), y además la $\mathrm{ET}_{0}$ fue de las más bajas de la serie. Por otro lado, 2005 fue un año poco lluvioso en los meses de mayores necesidades de riego. La variabilidad meteorológica y sobre todo las necesidades de riego de los meses de verano determinan los costes eléctricos que afectan a la productividad global de la zona.

En la Figura 3 se muestran para toda la zona y a escala de parcela: la distribución espacial de los suelos (Figura 3a), la distribución de cultivos utilizada para el análisis (Figura 3b), la producción media en porcentaje sobre el máximo rendimiento (Figura 3c) y dos parámetros de calidad del riego, eficiencia (Figura 3d) y uniformidad de reparto (Figura 3e). Los resultados de producción y calidad del riego corresponden a la campaña 2014.

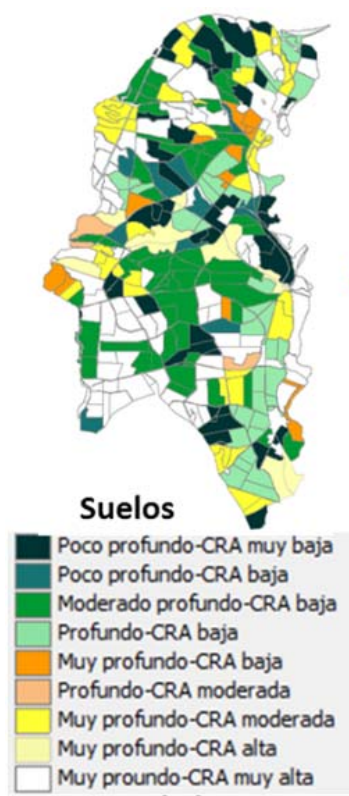

(a)

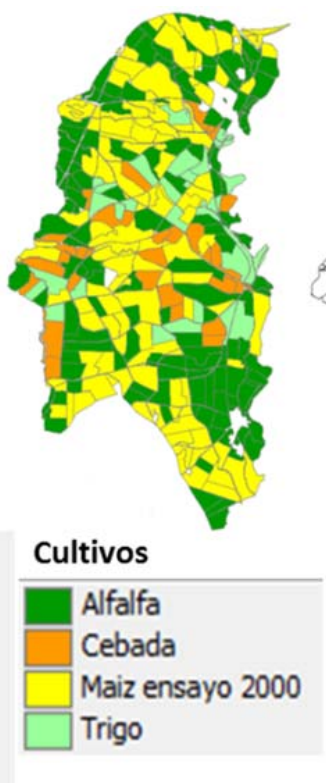

(b)

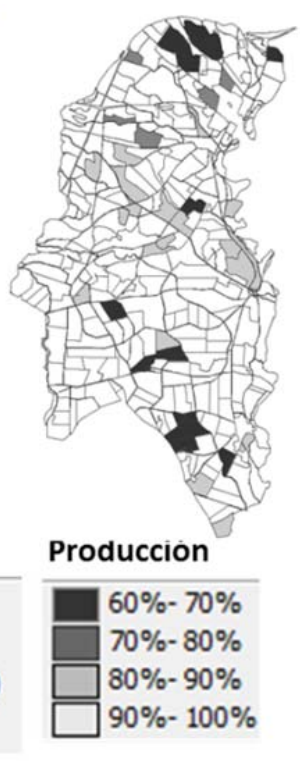

(c)

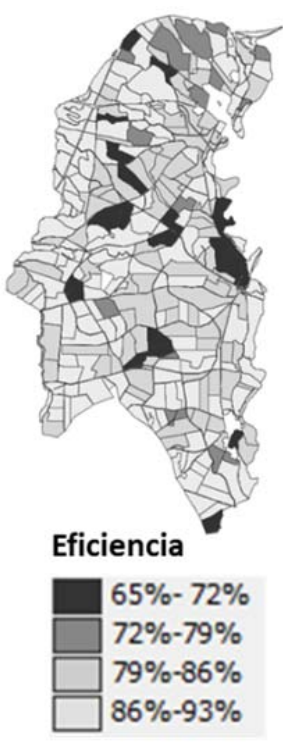

(d)

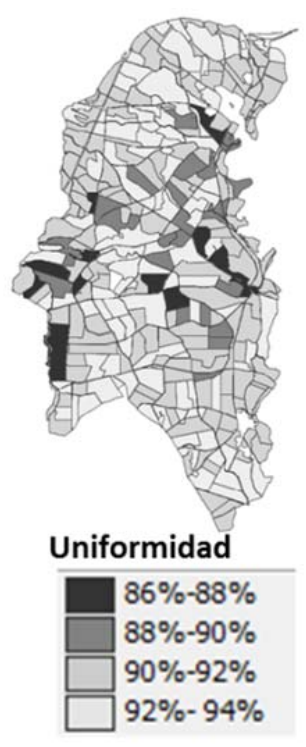

(e)

Figura 3. Suelos (a), alternativa de cultivo (b) en la zona de estudio, producción media \% (c) Eficiencia \% (d) y uniformidad \% (e), año 2014.

Se identificaron 9 tipos de suelos según su profundidad y CRA (Figura 3a). Los más abundantes, el $33 \%$ de superficie de la zona, son suelos muy profundos con una CRA muy alta, los suelos pobres desde el punto de vista hidráulico (poco profundos y CRA muy baja) aparecen en un 15\% de la superficie total de la CR. La distribución de los cultivos se realizó de forma aleatoria y se conservó esta misma distribución en todos los análisis realizados en este estudio.

En la Figura 4 se presenta la media por cultivo del consumo de agua (Figura 4a), la producción (Figura 4b), la eficiencia estacional del riego (Figura 4c) y la uniformidad estacional (Figura 4d) para el año 2014.

En cuanto al consumo de agua de riego, el consumo medio de la alfalfa fue de $8333 \mathrm{~m}^{3} \mathrm{ha}^{-1}$, con un CV entre parcelas del 5\%. El consumo del maíz fue de $6478 \mathrm{~m}^{3} \mathrm{ha}^{-1}$ con un CV del 5\%, el de la cebada $4069 \mathrm{~m}^{3} \mathrm{ha}^{-1}$, con un CV del $11 \%$ y el del trigo, $4509 \mathrm{~m}^{3}$ ha $^{-1}$ y un CV del $9 \%$.

En 2014 la producción media de alfalfa fue del 98\% con un CV de 2,6\%, la del maíz del $91 \%$ con mayor variabilidad entre parcelas, CV de 10,5\%, la de la cebada de $97 \%$ con un CV de $3,4 \%$ y la del trigo del $95 \%$ con un CV de 4,6\%. Los valores de producción media 
tanto de alfalfa como de cebada fueron elevados, indicando que los cultivos se regaron adecuadamente y también que, si en algún momento se retrasó la aplicación del riego a estos cultivos, la producción no se vio afectada. Sin embargo, la producción media del maíz fue menor y su variabilidad entre parcelas más elevada. Esto se debe a que los problemas que originaron retrasos en la aplicación del riego en maíz sí que afectaron al desarrollo y producción del mismo, pues se trata de un cultivo muy sensible al estrés hídrico. La distribución espacial de las producciones (Figura 3c) está muy relacionada con la distribución de suelos (Figura 3a) y con la de cultivos (Figura 3b). Las parcelas que presentan producciones más bajas, se corresponden con el cultivo de maíz y se localizan en suelos con baja calidad para el riego (suelos poco profundos y con una CRA muy baja). El riego de suelos de baja calidad hidráulica exige un manejo muy cuidadoso, como aplicar riegos ligeros con mayor frecuencia. Este manejo a veces no es posible en momentos de máxima demanda hídrica, ya que la disponibilidad de la red no permite aplicar el riego con la frecuencia que estos suelos requieren. Cuando estos suelos se combinan con cultivos de alta sensibilidad al estrés hídrico como el maíz, la producción de dichas parcelas se resiente (parcelas de colores más oscuros en la Figura 3c).
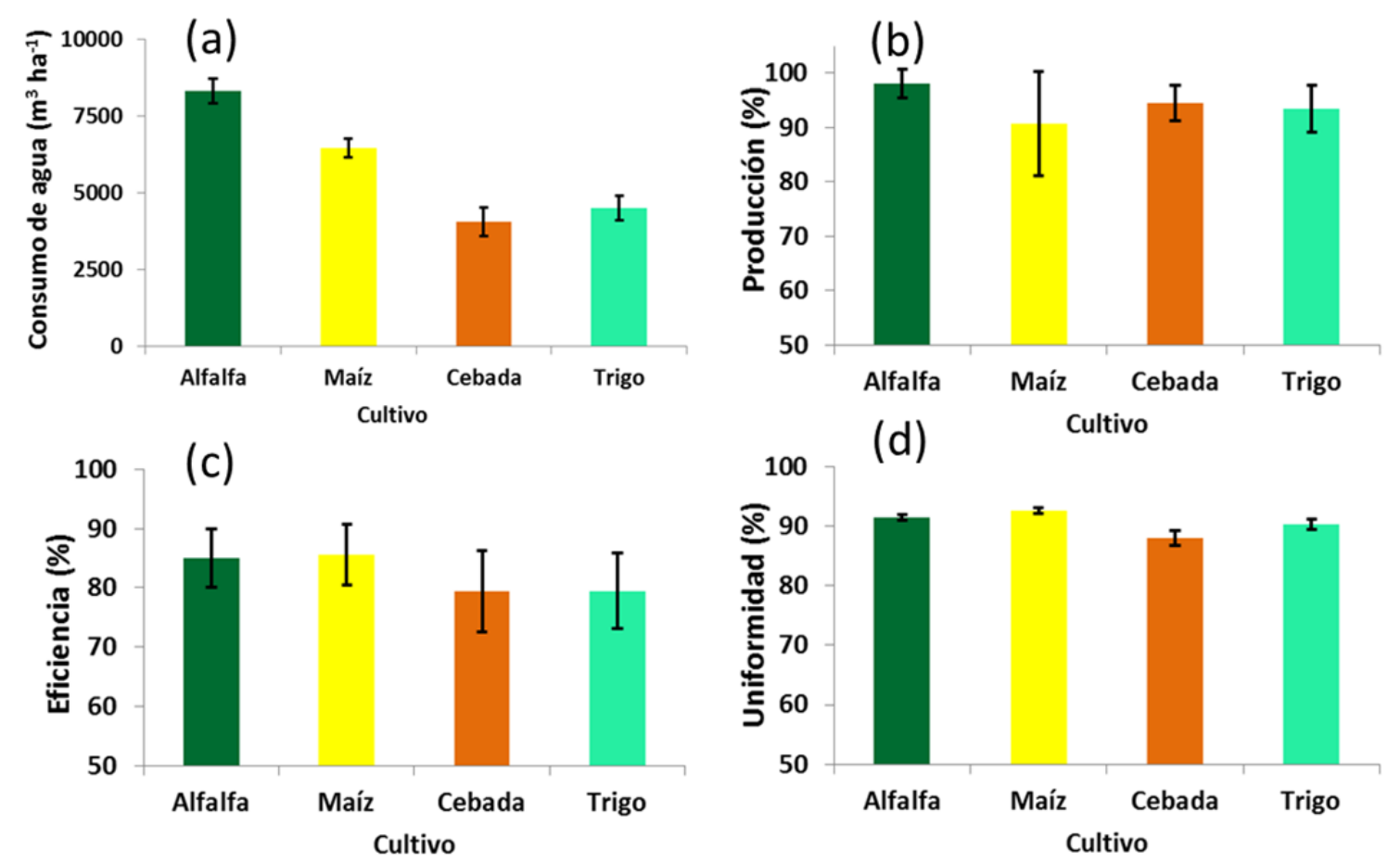

Figura 4. Consumo de agua (a) en $\mathrm{m}^{3} \mathrm{ha}^{-1}$, producción media \% (b), eficiencia \% (c) y uniformidad \% (d) por cultivo, resultados del 2014. Las barras indican la variabilidad entre las parcelas del mismo cultivo.

La eficiencia del riego media global durante la campaña 2014 fue del 84\%. La distribución espacial de dicha eficiencia (Figura 3d) indica que las parcelas que presentan menores eficiencias de riego se corresponden con las de los suelos más pobres desde el punto de vista hidráulico (poco profundos y CRA muy baja o baja). En estos suelos las pérdidas por percolación profunda fueron más importantes. El CU medio global en la campaña 2014 fue del 91\%. El análisis espacial de la uniformidad (Figura 3e) muestra parcelas con uniformidades inferiores al $90 \%$ que en la mayoría de los casos están cultivadas de cebada y de trigo. La baja uniformidad se justifica por el periodo de riego de estos cultivos que coinciden con los meses más ventosos del año, abril y mayo. La uniformidad del riego por aspersión se ve gravemente afectada por la velocidad del viento. 


\subsection{Análisis de alternativas de gestión y diseño de la zona regable}

En la Figura 5 se presenta la comparación entre las 4 alternativas de gestión y diseño analizadas en la zona: GRD, GRT $T_{\mathrm{DO}}, \mathrm{GRT}_{\mathrm{DM}}$ y GR2R en términos de consumo de agua (Figura 5a), ingresos brutos (Figura $5 b$ ), factura eléctrica (Figura $5 c$ ) e ingresos netos (Figura $5 \mathrm{~d}$ ). El consumo medio de agua en la gestión en turnos de presión (GRT $\mathrm{DO}_{\mathrm{DO}}$ y $\mathrm{GRT}_{\mathrm{DM}}$ ) fue inferior (entre un 3 y un 3,5\%) al consumo en una gestión a la demanda tanto en una red (GRD) o en dos redes (GR2R). Pero este menor consumo afectó de forma importante a la producción media de los cultivos y a sus ingresos brutos (Figura 5b). Las modificaciones en el diseño de la red para adaptarla a una gestión por turnos mejoraron los ingresos brutos respecto a los resultados de aplicar esta gestión en la red original, sin embargo, no se alcanzaron los ingresos brutos de la GRD. En cuanto a la GR2R, los ingresos brutos resultaron en valores medios muy similares a los de la GRD, y con menos variabilidad interanual ya que el CV no supera el 1,5\%.
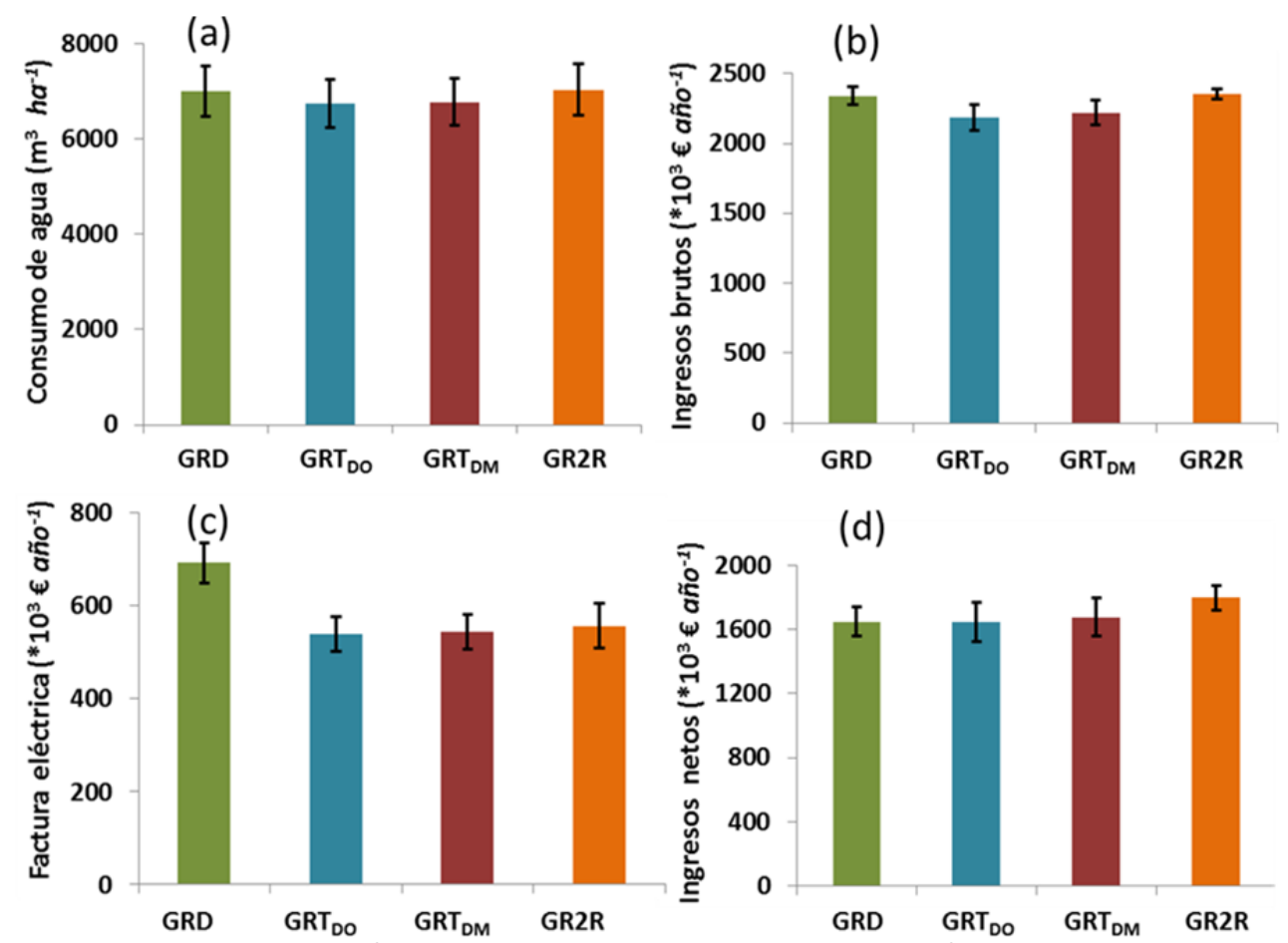

Figura 5. Ingresos brutos $\left({ }^{\star} 10^{3} €\right.$ por año), factura eléctrica $\left({ }^{\star} 10^{3} €\right.$ por año), ingresos netos $\left({ }^{\star} 10^{3} €\right.$ por año) y consumo de agua $\left(\mathrm{m}^{3} \mathrm{ha}^{-1}\right)$ para las 4 alternativas: GRD, GRT $T_{D O}, G R T_{D M} y$ GR2R. Las barras representan la variabilidad entre los años de estudio.

Como era de esperar (ya que el volumen de riego aplicado en notablemente inferior), la factura eléctrica del riego a turnos en sus dos modalidades (GRT $T_{D O}$ y $G R T_{D M}$ ) fue inferior (aproximadamente un 22\%) a la del riego a la demanda, especialmente el de toda la red GRD. Sin embargo, los valores medios de los ingresos netos (Figura $5 d$ ) en la gestión a turnos, $\mathrm{GRT}_{\mathrm{DO}}\left(1,649 \mathrm{M} €\right.$ por año) y $\mathrm{GRT}_{\mathrm{DM}}(1,677 \mathrm{M} €$ por año), son muy similares a los obtenidos en la GRD (1,650 M€ por año), lo que indica que lo que se ahorra en electricidad y se pierde en producción. La conclusión es que en esta red de riego el cambio de la gestión de riego a la demanda a riego organizado en turnos necesita un cambio importante de la red y que las mejoras en productividad global no son muy relevantes. La división de la zona en dos redes de riego a la demanda independientes (GR2R) mantiene los ingresos brutos respecto a la GRD y permite un ahorro en la factura eléctrica (20\%), lo que se traduce en una mejora de los ingresos netos del 9\% (1,797*M€ por año) comparando a la GRD. Esta división de la red permite, también, una amortiguación del efecto de la variabilidad meteorológica interanual sobre les ingresos netos ya que el CV es de 4,2\% en la GR2R frente a 5,6\% en la GRD, 7,4\% en la GRT 
Para analizar la disminución de los ingresos brutos de la GRT frente a la GRD se analizaron los resultados de las producciones medias por parcela (Figura 6) para el año 2014. En la Figura 6a se presentan los suelos, en la Figura $6 \mathrm{~b}$ los cultivos, en la Figura $6 \mathrm{c}$ la producción en \% de la GRD, en la Figura 6d la producción en \% de la GRTDM y en la Figura 6d la producción en \% de la GR2R. En general las parcelas con menores producciones (colores más oscuros) en la gestión a la demanda también resultan en producciones menores en la gestión a turnos. Sin embargo en la gestión a turnos el número de parcelas con problemas de producción es mayor, y los valores de producción son en general inferiores. En la mayoría de los casos se trata de parcelas que o bien tienen un suelo pobre (poco profundo y CRA baja o muy baja, colores verde oscuros en la Figura 6a), o/y presentan un número elevado de sectores/posturas de riego, o/y pertenecen a la subzona (turno) alta o/y están cultivadas de maíz, un cultivo sensible al estrés hídrico

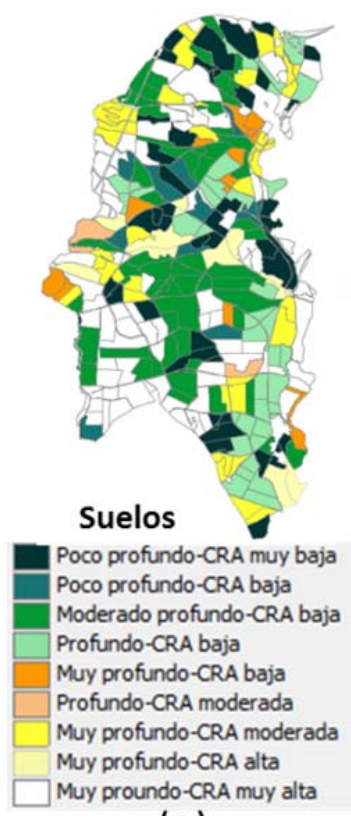

(a)
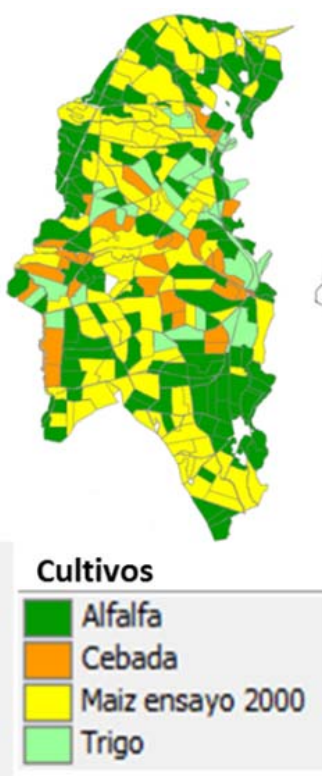

(b)

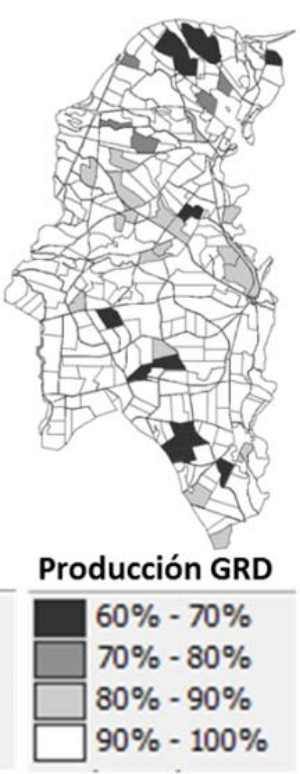

(c)

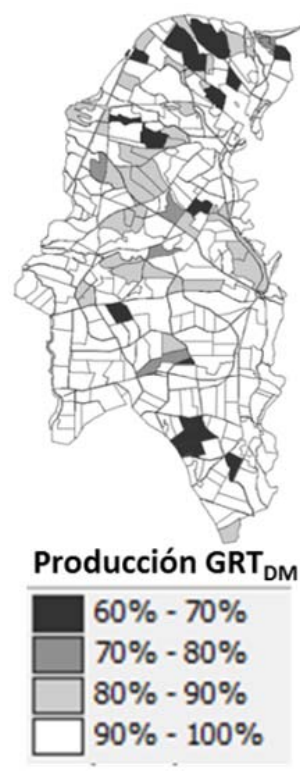

(d)

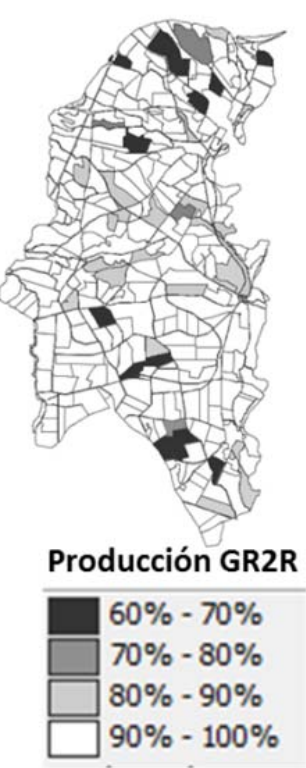

(e)

Figura 6. Producción media (\%) de los hidrantes para las alternativas: $G R D, G R T_{D M}$ y GR2R, año 2014.

\section{Conclusiones}

Este estudio presenta una aplicación de la herramienta de simulación CINTEGRAL que permite analizar el diseño y la gestión de una zona regable adaptándose a las redes (colectivas y parcelarias), los suelos, la meteorología, la agronomía, así como a los precios de cosecha y de los insumos de producción (entre ellos el agua y la energía). Este enfoque permite realizar un análisis global que incorpora la realidad agraria local de la zona regable.

Los resultados obtenidos en una gestión a la demanda muestran el efecto de la variabilidad meteorológica interanual sobre los ingresos brutos, ingresos netos y la factura eléctrica. Las producciones mínimas se registran en suelos más pobres desde el punto de vista hidráulico (poco profundos y CRA muy baja o baja) y para cultivos más sensibles al estrés hídrico (maíz).

La comparación de las 4 alternativas, gestión a la demanda de la zona global, gestión en 2 turnos de riego adaptados a zonas de presión homogénea (sin y con cambios en el diseño) y gestión en 2 redes independientes, muestra que la división de la zona en dos redes permite reducir la factura eléctrica en $20 \%$ sin afectar a la producción, lo que arroja un incremento del $9 \%$ de los ingresos netos. Sin embargo, la gestión a turnos reduce la factura eléctrica pero también produce notables mermas de producción que reducen los ingresos brutos, resultando en un incremento de los ingresos netos en un escaso $2 \%$ respecto a la gestión a la demanda. 


\section{Referencias}

Alexandratos, N. and Bruinsma, J., (2012), World Agriculture: Toward 2030/2050, The 2012 Revision. Publicación FAO: 12-03.

Carrillo Cobo, M.T., Rodríguez Díaz, J.A., Montesinos, P., López Luque, R., Camacho Poyato, E., (2011). Low energy consumption seasonal calendar for sectoring operation in pressurized irrigation networks. Irrig Sci 29:157-169.

Dechmi, F., Playán, E., Cavero, J., Martinez-Cob, A., Faci, J.M., (2004a). Coupled crop and solid-set sprinkler simulation model. I: Model development. J Irrig Drain Eng ASCE 130(6):499-510.

Fernández García, I., Moreno, M.A., Rodríguez Díaz, J.A., (2014). Optimum pumpingstation management for irrigation networks sectoring: case of Bembezar MI (Spain). Agric. Water Manage. 144, 150-158.

Fukui, Y., Nakanishi, K., Okamura, S., (1980). Computer evaluation of sprinkler irrigation uniformity. Irrig. Sci. 2, 23-32.

Martínez-Cob, A., Zapata, N., Sánchez, I., (2010). Viento y riego: la variabilidad del viento en Aragón y su influencia en el riego por aspersión, Fernando el Católico.

Nogüés, J., (2002). Mapa de suelos (E 1/25000) de Barbués y Torres de Barbués. Aplicación para modernización de regadíos, Consejo de Protección de la Naturaleza de Aragón.

Playán, E., Zapata, N., Faci, J. M., Tolosa, D., Pelegrín, J., Salvador, R., Lafita, A., Sánchez, I. (2006). Assessing sprinkler irrigation uniformity using a ballistic simulation model. Agric. Wat. Manage., 84 (1-2):89-100.

Rossman, L.A., Clark, R.M., Grayman, W.M., (1994). Modeling chlorine residuals in drinkingwater distribution-systems

Smith, M., (1992). CropWat: a computer program for irrigation planning and management. FAO Irrig and Drain paper 46, Rome, Italy.

Zapata, N., Latorre, B., Citoler, F.J., Gallinat, J., Bescós, M., Castillo, R., Mantero, N., Burguete, J. and Playán, E. Collective irrigation network design and management for energy optimization: the "cintegral" tool. ICID 26th Euro Mediterranean Regional Conference and ICID 56th International Executive Council. Innovate to Improve Irrigation Performance. Montpellier, France. 11-16 october 2015.

Zapata, N., Playán, E., Skhiri, A., Burguete, J., (2009). A collective solid-set sprinkler irrigation controller for optimum water productivity. J Irrig Drain Eng ASCE 135(1):13-24. 